\title{
B7-H4 overexpression contributes to poor prognosis and drug-resistance in triple-negative breast cancer
}

Ling Wang ${ }^{1 *+}$ (D) Chao Yang ${ }^{2}$, Xin-bo Liu ${ }^{3}$, Li Wang $^{4}$ and Fu-biao Kang ${ }^{{ }^{*}+}$

\begin{abstract}
Background: The expression of the immunoregulatory protein $\mathrm{B} 7-\mathrm{H} 4$ has been reported in many types of cancer, including breast cancer. However, its role in triple-negative breast cancer (TNBC), especially its correlation with patients' prognosis and chemoresistance remains unclear.

Methods: The expression of B7-H4 in TNBC tissues and cell lines were measured with Real-Time PCR and western blotting. 65 cases of TNBC tissue samples and adjacent non-tumor tissue samples were analyzed by immunochemistry to demonstrate the correlation between the B7-H4 expression and clinicopathological characteristics. In vitro studies assessed mAb MIH43 alone and in combination with transfecting B7-H4 siRNA on the growth of chemosensitive and chemoresistant TNBC cell lines by CCK-8 and apoptotic enzyme-linked immunosorbent assay (ELISA).

Results: B7-H4 expression was detected positive in 59 of 65 (90.8\%) different stage TNBC patients, especially in the samples of recurrence TNBC patients after receiving neoadjuvant chemotherapy treatment. Survival curves showed that patients with $\mathrm{B} 7-\mathrm{H} 4$ overexpression had significantly shorter survival and recurrence time than those with low B7-H4 expression $(p<0.005)$. Univariate and multivariate COX regression analysis demonstrated that B7-H4 was an independent predictor for advanced tumor stage. The monoclonal antibody of B7-H4 has the potential anti-proliferative effects on inhibiting the chemoresistant TNBC cell lines and increasing the sensitivity of TNBC cell lines to doxorubicin, paclitaxel or carboplatin. RNAi-mediated silencing of B7-H4 in TNBC cells enhanced drug-induced apoptosis via inhibiting PTEN/PI3K/AKT pathway, whereas reexpression of B7-H4 in B7-H4 knockdown and low B7-H4 expressing cells increased the phosphorylation of PI3K and AKT along with restoration of PETN expression.
\end{abstract}

Conclusions: Our data show that B7-H4 is a biomarker indicative of a poor prognosis in TNBC patients and at least partially downregulated in chemoresistance via PTEN/PI3K/AKT pathway. Targeting B7-H4 might provide an attractive therapeutic approach specifically for TNBC patients.

Keywords: Triple-negative breast cancer, B7-H4, Proliferation, Apoptosis, Chemoresistance

\footnotetext{
*Correspondence: wangling8106@163.com; kangfb@hotmail.com

${ }^{\dagger}$ Fu-biao Kang and Ling Wang are Co-Corresponding authors.

1 Department of Orthopedic Oncology, the Third Hospital of Hebei

Medical University, Shijiazhuang, Hebei, People's Republic of China

${ }^{5}$ Department of Liver Diseases, Bethune International Peace Hospital,

Shijiazhuang, Hebei, People's Republic of China

Full list of author information is available at the end of the article
} 


\section{Background}

Breast cancer is a heterogeneous disease and TNBC is one of the most biologically aggressive subtypes [1, $2]$. TNBC represents $10-20 \%$ of breast cancer and the mainstay of therapy remains chemotherapy and no targeted therapy is currently available for it. Although TNBC patients are sensitive to adjuvant chemotherapy, the prognosis is very poor and prone to cause chemotherapy-resistant and recurrence within 3-years [3, 4]. Therefore, to identify potential therapeutic targets, a better understanding of the biology of TNBC is needed. There is increasing evidence showed that adding targeted therapies to adjuvant chemotherapy may increase the sensitivity of residual disease, which will reduce the dose of chemotherapy necessary to kill the remaining tumor cells, thereby minimizing the toxicity of the prolonged treatment $[5,6]$.

Upregulation of immune inhibitory molecules such as co-regulatory ligands/receptors and tolerogenic enzymes by cancer cells allow tumors escape from immune attack $[7,8]$. Immune checkpoint inhibitors such as PD-1 and CTLA-4 have shown prominent and durable responses in diverse malignancies $[9,10]$. B7- $\mathrm{H} 4$ is one of the most recently identified members of the $B 7$ homologue family of immune co-regulatory molecules and shown to exert an immunosuppressive effect in regulation of $\mathrm{T}$ cell immunity through the inhibition of T-cell function, such as activation, proliferation, cytokine production and cytotoxic activity [11-13]. Recently, many studies have reported that $\mathrm{B} 7-\mathrm{H} 4$ is implicated in various types of human tumors, including renal cell carcinoma, ovarian cancer, gastric cancer, and breast cancer, where it plays an important part in tumor progression and is associated with a poor prognosis [14-17]. However, whether and how the engagement of $\mathrm{B} 7-\mathrm{H} 4$ by counter molecules affects the fate of B7-H4-expressing cells is poorly understood.

In this study, we investigate the prevalence and prognostic value of B7-H4 on TNBC with the clinicopathological characteristics and patients' outcome. In addition, we also demonstrate the potential role of $\mathrm{B} 7-\mathrm{H} 4$ played in TNBC cells after received chemotherapy treatment. The aim of our study was to further clarify whether B7-H4 could be identified as a reliable marker for the appropriate selection of high-risk patients eligible for personaldesigned targeted therapeutic agents. And whether blocking B7-H4 could be an alternative approach to avoid residual cancer cells become drug-resistant.

\section{Materials and methods}

\section{Microarray data sources}

Microarray datasets of invasive breast carcinoma (IDC) were downloaded from the Cancer Genome Atlas:
Invasive Breast Carcinoma Gene Expression Data, 2011, (http://tcga-data.nci.nih.gov/tcga/). These data were accessed via the ONCOMINE Cancer Profiling Database and used to investigate $\mathrm{B} 7-\mathrm{H} 4$ expression in IDC and non-IDC of breast cancer.

\section{Patients and diagnostic criteria}

Tissue samples (frozen and FFPE) were separately obtained from 65 untreated Chinese women diagnosed with TNBC at the Fourth Hospital of Hebei Medical University from January 2005 to December 2010. All cases were reviewed by two experienced pathologists, and assessed in accordance with the criterion of the world health organization (WHO) breast cancer pathology [18]. In our study, TNBC diagnosis was determined and based on negative test results in ER, PR, and HER-2 from biopsy samples. The baseline clinicopathological data including age, tumor size, lymph node metastasis, distant metastasis, tumor grade, TNM stage, histological type, and survival information were retrieved. The TNM stage was determined based on the American Joint Committee on Cancer (AJCC) criteria and the histological grade was assessed according to the modified Bloom-Richardson classification. For these recurrence patients, the initial symptoms for patients are different, including neurological symptoms, abdominal symptoms, lung symptoms and bone symptoms, etc. Some patients discover the recurrence by themselves, while others were detected by physician examination. Breast cancer recurrence was diagnosed by radiography or discovered by tumor CA 15-3 elevation or/and CAE elevation in patients. This study was reviewed and approved by the Review Board of Fourth Hospital of Hebei Medical University. All patients gave their written and informed consent.

\section{Cell lines and cell culture}

TNBC cell lines MDA-MB-231, MDA-MB-435, MDAMB-468 and non-TNBC cells MCF-7 and MCF-10F were obtained from the Cell Bank of the Chinese Academy of Sciences (Shanghai, China) and cultured according to the instructions from American Type Culture Collection (ATCC). Cell lines were all maintained in suitable medium supplemented with $10 \%$ fetal bovine serum and $1 \%$ penicillin/streptomycin. For MDA-MB-435/DOX (doxorubicin) cell culture, DOX was added in the RPMI 1640 medium to achieve the final DOX concentration of $2 \mu \mathrm{g} / \mathrm{mL}$ [19]. All cell lines were maintained in antibioticfree medium at $37{ }^{\circ} \mathrm{C}$ in a $5 \% \mathrm{CO}_{2}$ atmosphere and routinely screened for mycoplasma contamination.

\section{Immunohistochemical (IHC) staining}

For all IHC analyses, FFPE tumor samples were prepared for $5 \mu \mathrm{m}$ slices and antigen retrieval was performed by 
citrate buffer solution $(\mathrm{pH}=6)$ for $5 \mathrm{~min}$. Endogenous peroxidase was blocked with $0.3 \% \mathrm{H}_{2} \mathrm{O}_{2}$ in methanol for $15 \mathrm{~min}$, and all slides were heated to $100{ }^{\circ} \mathrm{C}$ for $20 \mathrm{~min}$ and then cooled at room temperature. Non-specific binding sites were blocked by $10 \%$ bovine serum albumin (BSA) for $30 \mathrm{~min}$. The slides were then washed in phosphate-buffered saline, and primary antibody against B7-H4 (diluted in 1:500) was applied for overnight incubation. On the next day, anti-mouse/rabbit IgG were added for $1 \mathrm{~h}$ incubation at $37{ }^{\circ} \mathrm{C}$. Color development with $\mathrm{DAB}$ substrate was performed and counterstaining with hematoxylin was conducted.

\section{Evaluation of immunohistochemical staining}

Evaluation of B7-H4 staining in tumor cells was evaluated by authorized pathologists who had no knowledge of the patients' clinical status and outcome. B7-H4 expression scores were given separately for the stained area and for the intensity of staining. Quantification was made as follows; $\leq 25 \%$ of the cancer cells: $1,>26 \%$ to $\leq 50 \%$ of the cancer cells: $2,>51 \%$ to $\leq 75 \%$ cancer ce cancer cells: 3 , $>76 \%$ of the cancer cells: 4 ; intensity of staining: absent/ weak: 1 , moderate: 2 , strong: 3 . Each section had a final grade that derived from the multiplication of the area and intensity scores. The final B7-H4 staining score was calculated using the percent of positive cell score $\times$ staining intensity score ranging $0-12$. The final scores $\leq 4$ was classified as tumors with low B7-H4 expression, whereas sections with a final score of $>4$ were classified as tumors with high B7-H4 expression.

\section{In vitro growth inhibition}

Cells $\left(1 \times 10^{4}\right.$ cells $)$ were initially plated in triplicate in 96-well culture plates. Twenty-four hours later, the medium was replaced with fresh medium with or without different drugs and incubated for indicated time. Cell viability was determined by CCK-8. In order to detect the effects of $\mathrm{B} 7-\mathrm{H} 4$ in cell proliferation, $\mathrm{B} 7-\mathrm{H} 4 \mathrm{mAb}$ (MIH43, ab110221) and its isotype control mAb IgG1 (RM106, ab190481) were purchased from abcam company and perform the cell growth assay. The absorbance value at $450 \mathrm{~nm}$ was read using a microplatereader (BioRad, CA, USA).

\section{Transient transfection of B7-H4 overexpressing or silencing plasmid}

To further analyze the role of $\mathrm{B} 7-\mathrm{H} 4$ in TNBC, we transfected TNBC cells with the B7-H4 cDNA (Origene, Inc) or B7-H4 siRNA (Origene, Inc) using Lipofectamine ${ }^{3000}$ (Invitrogen, CA). In brief, about $3 \times 10^{5}$ cells were seeded per well in a 6 well plate. After $24 \mathrm{~h}$, the cells were transfected with $1.5 \mu \mathrm{g}$ of cDNA or siRNA plasmid for $6 \mathrm{~h}$, and the media were replaced with fresh growth medium. At
$48 \mathrm{~h}$ after transfection, cells were harvested for analysis. The silencing or overexpressing effects of $\mathrm{B} 7-\mathrm{H} 4$ in TNBC cells were detected and confirmed (Additional files 1, 2).

RNA extraction, reverse transcription and real-time RT-PCR Total RNA was extracted from cell lines and freshly frozen samples with TRIzol reagent (Invitrogen, USA) and was reverse-transcribed with the first strand cDNA synthesis kit (Invitrogen). Real-time PCR reactions were conducted using SYBR Premix Ex Taq II (Takara). Reverse transcriptase was used as the negative control, and glyceraldehyde-3-phosphate dehydrogenase (GAPDH) was used as the endogenous control. All experiments were repeated three times. The PCR primers used in this study were as follows: B7-H4 (F5': AGGGAG TGGAGGAGGATACAG, R5': GCAGCAGCCAAAGAG ACAG), GAPDH (F5': CACCATCTTCCAGGAGCGAG, R3': GACTCCACGACGTACTCAGC).

\section{Quantification of apoptosis by ELISA kit}

An apoptosis ELISA kit (Roche Diagnostics Co.) was used to quantitatively measure cytoplasmic histoneassociated DNA fragments. After treatment with different concentration of DOX or B7-H4 mAb MIH43 (5 or $10 \mu \mathrm{g} / \mathrm{mL}$ ) up for $72 \mathrm{~h}$, cells were analyzed by following manufacturer's protocol. Each experiment was repeated three times.

\section{Western blot analysis}

Protein from TNBC tissue samples and cells were separated by SDS-PAGE and then electro-transferred onto nitrocellulose membrane (Bio-Rad). The primary antibodies used included antibodies to B7-H4, PARP, Caspase-3, Caspase-7, Caspase-9, PETN, p-PI3K, PI3K, p-AKT, AKT and GAPDH were purchased from Abcam company. Membranes were probed with indicated antibodies by following the manufacturer's protocol, and immunoreactive bands were visualized by using ECL Western Blotting Substrate (Pierce Biotechnology, Inc.). Each experiment was repeated three times.

\section{Statistical analysis}

Results are reported as mean \pm SD. All the experimental data were analyzed by the SPSS 20.0 statistical software package. The Mann-Whitney $U$ test, $X^{2}$ test, Pearson Chi square test or Spearman rho test were performed for comparative statistical evaluations among groups and for correlation analysis with histological and clinical parameters (age, gender, tumor stage, tumor grade, and postoperative survival). Survival periods were counted in months from the date of first visit to date of death or last 
follow-up before study closure. We used Kaplan-Meier method to estimate the overall survival for low and high levels of B7-H3 expression. A $p$ value $<0.05$ was considered as statistically significant.

\section{Results}

\section{Elevated B7-H4 expression associated with human TNBC progression}

To evaluate the expression of $\mathrm{B} 7-\mathrm{H} 4$ in human TNBC, we used the publicly available cancer microarray database to estimate the status of $\mathrm{B} 7-\mathrm{H} 4$ transcript. The result demonstrated differential B7-H4 expression was significantly increased in invasive breast carcinoma compared with non-invasive breast carcinoma tissues (Fig. 1a). To further testify the analytical result, 10 fresh TNBC tissue and adjacent non-tumor adjacent tissue (NAT) together with TNBC cell lines were also collected. Following RNA extraction, qRT-PCR confirmed the high presence of B7-H4 mRNA in all these TNBC samples with transcript levels, and MDA-MB-435 cells showed baseline high B7-H4 expression among all the tested cells (Fig. 1b, c).

In addition to the transcript level of B7-H4 expression, we next evaluated B7-H4 expression at the protein level via immunohistochemical staining. Figure $1 \mathrm{~d}$ results showed that B7-H4 expression was detected positive in 59 of $65(90.8 \%)$ patients with different stage TNBC who had not received neoadjuvant chemotherapy. In all positive cases, $\mathrm{B} 7-\mathrm{H} 4$ expression was present diffusely throughout the cytoplasm with a pronounced membranous component. According to the staining intensity, there were $6(9.2 \%)$ cases with no tumor B7-H4 intensity, 7 (10.8\%) cases with weak tumor B7-H4 intensity, 25 (38.5\%) with moderate intensity, and with 27 (41.5\%) marked intensity. According to their positive immunoreactivity staining area, a total of $83.1 \%$ of tumor samples were high B7-H4 expression, while $16.9 \%$ showed a lower degree of B7-H4 staining. However, there was limited expression of $\mathrm{B} 7-\mathrm{H} 4$ in normal breast tissues.

Pertinent clinicopathological findings of the enrolled patients are summarized in Table 1. High expression level of B7-H4 in TNBC was more common in IDC compared with non-IDC subtype and was associated with advanced TNM stage $(p=0.006)$ and likely to develop metastasis $(p=0.001)$ and recurrence $(p=0.003)$. However, $\mathrm{B} 7-\mathrm{H} 4$ expression was not associated with age $(p=0.612)$, menopausal status $(p=0.425)$, tumor size $(p=0.055)$ histological subgroups $(p=0.051)$. Surprisingly, we noticed that there was a reverse correlation between the expression of $\mathrm{B} 7-\mathrm{H} 4$ and androgen receptor $(\mathrm{AR}, \mathrm{r}=-0.317$, $p=0.004)$. Taken together, these data confirmed that B7-H4 expression might be functionally important in tumor progression and metastasis in TNBC.
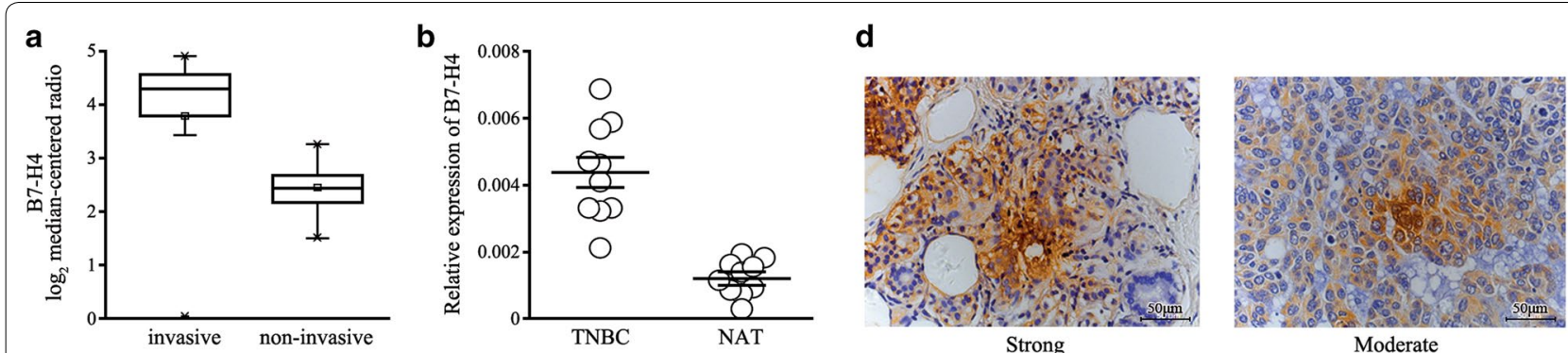

C
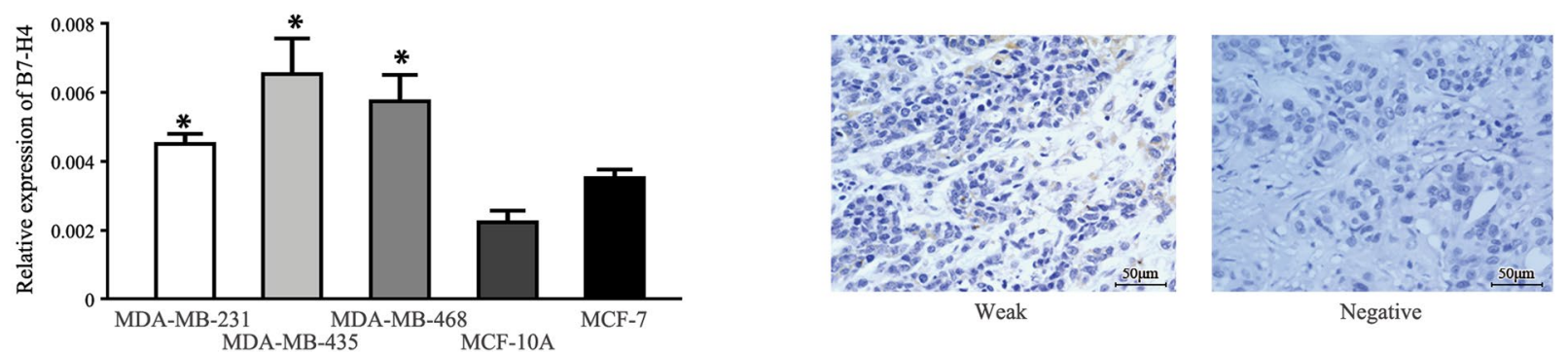

Fig. 1 B7-H4 is highly expressed in invasive breast cancer and particularly overexpressed at high levels in TNBC subtype. a B7-H4 is significantly overexpressed in invasive breast cancer tissue compared with non-invasive breast cancer tissue samples from the public breast cancer microarray database. $\mathbf{b}$ The expression level of B7-H4 mRNA in TNBC samples compared with adjacent non-tumor tissue samples. $\mathbf{c}$ The expression level of B7-H4 mRNA in various breast cancer cell lines was evaluated using real-time PCR. The data are presented as the mean \pm SD. $\mathbf{d}$ B7-H4 immunostaining in TNBC tissues and normal breast tissue. $\times 200$ magnification 
Table 1 Correlation between B7-H4 expression and clinicopathological parameters in TNBC

\begin{tabular}{|c|c|c|c|c|c|c|c|c|}
\hline \multirow[t]{2}{*}{ Parameters } & \multirow[t]{2}{*}{$\mathrm{n}$} & \multicolumn{2}{|c|}{ B7-H4 expression } & \multirow[t]{2}{*}{$p$} & \multicolumn{3}{|c|}{ B7-H4 intensity } & \multirow[t]{2}{*}{$p$} \\
\hline & & Low & High & & Weak & Moderate & Strong & \\
\hline \multicolumn{9}{|l|}{ Age } \\
\hline$\leq 50$ & 30 & 5 & 25 & \multirow[t]{2}{*}{0.612} & 4 & 14 & 12 & \multirow[t]{2}{*}{0.664} \\
\hline$>50$ & 29 & 5 & 24 & & 3 & 11 & 15 & \\
\hline \multicolumn{9}{|l|}{ Menopausal } \\
\hline Premenopausal & 30 & 6 & 24 & \multirow[t]{2}{*}{0.425} & 5 & 13 & 12 & \multirow[t]{2}{*}{0.440} \\
\hline Postmenopausal & 29 & 5 & 24 & & 2 & 12 & 15 & \\
\hline \multicolumn{9}{|l|}{ Tumor histology } \\
\hline IDC & 45 & 10 & 35 & \multirow[t]{2}{*}{0.051} & 7 & 21 & 17 & \multirow[t]{2}{*}{0.059} \\
\hline Non-IDC & 14 & 1 & 13 & & 0 & 4 & 10 & \\
\hline \multicolumn{9}{|l|}{ Tumor size } \\
\hline$\leq 2$ & 4 & 3 & 1 & \multirow[t]{3}{*}{0.055} & 1 & 2 & 1 & \multirow[t]{3}{*}{0.113} \\
\hline $2-5$ & 42 & 7 & 35 & & 6 & 20 & 16 & \\
\hline$>5$ & 13 & 0 & 13 & & 0 & 3 & 10 & \\
\hline \multicolumn{9}{|l|}{ Tumor grade } \\
\hline$|-| \mid$ & 26 & 9 & 17 & \multirow{2}{*}{0.002} & 7 & 12 & 7 & \multirow[t]{2}{*}{0.004} \\
\hline III & 33 & 1 & 32 & & 0 & 13 & 20 & \\
\hline \multicolumn{9}{|l|}{ TNM stage } \\
\hline I & 3 & 3 & 0 & \multirow[t]{3}{*}{0.006} & 1 & 2 & 0 & \multirow[t]{3}{*}{0.003} \\
\hline$\|$ & 41 & 7 & 34 & & 6 & 22 & 13 & \\
\hline III & 15 & 0 & 15 & & 0 & 1 & 14 & \\
\hline \multicolumn{9}{|l|}{ Metastasis } \\
\hline Yes & 21 & 9 & 12 & \multirow[t]{2}{*}{0.001} & 7 & 10 & 4 & \multirow[t]{2}{*}{0.001} \\
\hline No & 38 & 2 & 36 & & 0 & 15 & 23 & \\
\hline Ki-67 & & & & & & & & \\
\hline+ & 8 & 4 & 4 & 0.053 & 3 & 3 & 2 & 0.062 \\
\hline++ & 29 & 6 & 23 & & 4 & 13 & 12 & \\
\hline+++ & 22 & 2 & 20 & & 2 & 7 & 13 & \\
\hline$A R$ & & & & & & & & \\
\hline- & 38 & 3 & 35 & 0.007 & 2 & 11 & 25 & 0.003 \\
\hline+ & 21 & 9 & 12 & & 6 & 10 & 5 & \\
\hline Recurrence & & & & & & & & \\
\hline Yes & 25 & 9 & 16 & 0.003 & 7 & 10 & 8 & 0.002 \\
\hline No & 34 & 4 & 30 & & 2 & 13 & 19 & \\
\hline
\end{tabular}

Numbers in italic indicate statistical significance

\section{Elevated B7-H4 expression correlated with poor survival of human TNBC patients}

Cumulative survival time analyzed by Kaplan-Meier method showed that patients with high B7-H4 expression had significantly shorter survival times $(p=0.002$, Fig. 2a), 24 (48.9\%) of TNBC patients with high B7-H4 expression died at the time of study, compared with 1 (9.1\%) TNBC patients with low B7-H4 expression after diagnosis $(p=0.003)$. Moreover, the average time to recurrence was 48.1 months for patients with low B7-H4 expression, compared to 27.7 months for patients with high B7-H4 expression $(p=0.014)$.
In univariate analysis, larger tumor size, lower tumor grade, advanced TNM stage, high Ki-67, AR, B7-H4 expression, B7-H4 intensity and lymph node positivity were associated with shorter overall OS, while other clinical pathological characteristics, such as age, histological subtype did not influence the prognosis. In the multivariate analysis, $\mathrm{B} 7-\mathrm{H} 4$ staining intensity is an independent prognostic factor for OS in TNBC patients (RR: 0.313, 95\% CI 0.114-0.857, $p=0.024)$. Results from the univariate analysis and final multivariate Cox regression model are presented in Table 2. Therefore, these data suggested that weaker B7-H4 intensity is correlated with better prognosis or survival in TNBC. 

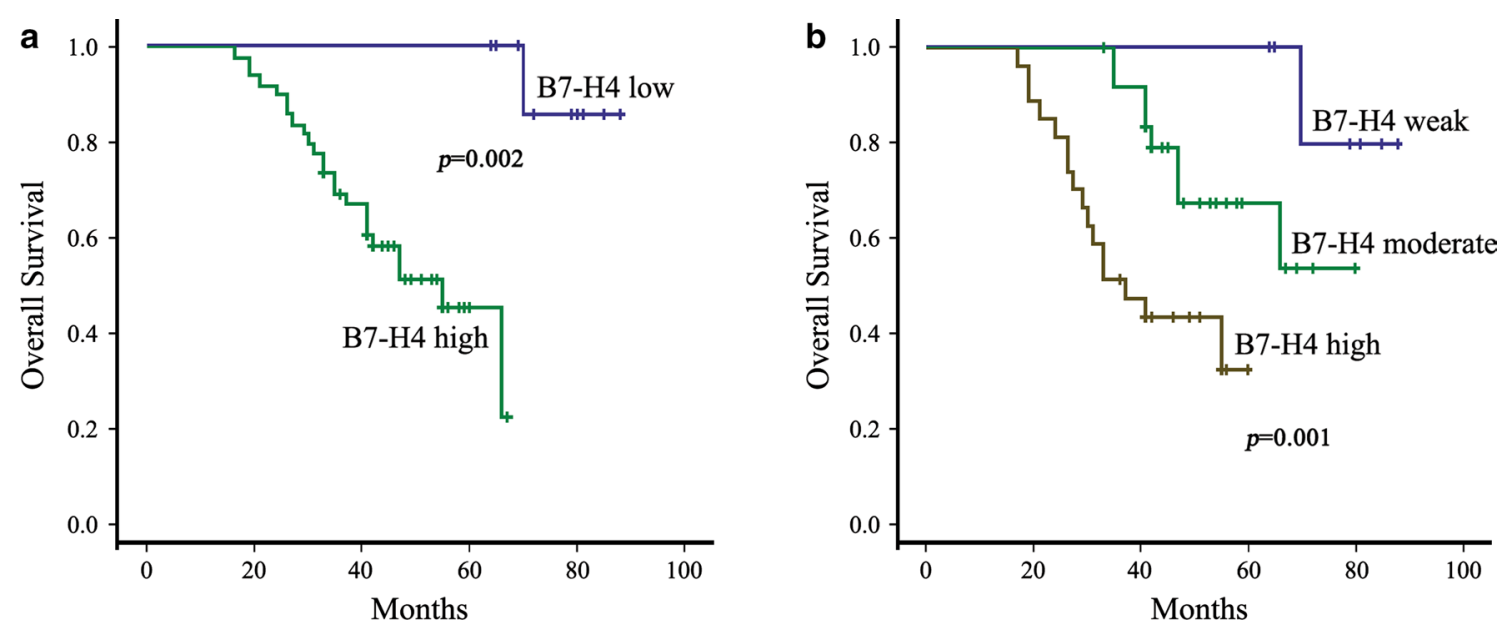

Fig. 2 Overall survival of 65 TNBC patients stratified by B7-H4 protein expression levels. a Kaplan-Meier plots of overall survival in TNBC patients from the whole data sets stratified by B7-H4 expression levels. b Kaplan-Meier plots of overall survival in TNBC patients from the whole data sets stratified by B7-H4 expression intensity

Table 2 Univariable and multivariable analysis for of TNBC overall survival

\begin{tabular}{lllll}
\hline Characteristics & Univariate & & Multivariate \\
\cline { 2 - 3 } & HR (95\% Cl) & $\boldsymbol{p}$ value & HR (95\% Cl) & $0.393(0.118-1.311)$ \\
\hline Tumor size & $2.418(1.118-5.228)$ & 0.025 & $0.316(0.041-2.446)$ & 0.314 \\
Tumor grade & $7.464(2.493-22.350)$ & 0.011 & $0.290(0.084-0.998)$ & 0.270 \\
TNM stage & $4.433(1.947-10.093)$ & 0.032 & $5.795(1.245-20.964)$ & 0.041 \\
Metastasis & $3.579(1.299-9.862)$ & 0.026 & $0.250(0.064-0.981)$ & 0.025 \\
Ki-67 & $4.630(2.106-10.178)$ & 0.022 & $0.374(0.058-2.414)$ & 0.138 \\
AR & $0.139(0.040-0.487)$ & 0.002 & $0.033(0.003--0.326)$ & 0.003 \\
Recurrence & $16.473(3.788-71.646)$ & 0.002 & $0.177(0.012-2.001)$ & 0.213 \\
B7-H4 (expression) & $0.122(0.022-1.012)$ & 0.045 & $0.313(0.114-0.857)$ & 0.024 \\
B7-H4 (intensity) & $3.837(1.773-8.307)$ & 0.007 &
\end{tabular}

Numbers in italic indicate statistical significance

\section{B7-H4 abnormally overexpressed on the samples of recurrence TNBC patients after neoadjuvant chemotherapy treatment}

Interestingly, we found out that there was a positive correlation between the expression status of $\mathrm{B} 7-\mathrm{H} 4$ and patients' recurrence except all the previous collected results. Many included studies showed that neoadjuvant treatment might differentially affect the patterns of recurrence and overall survival in TNBC patients [20]. Therefore, we analyzed the potential $\mathrm{B} 7-\mathrm{H} 4$ function in patients stratified by the likelihood of their cancer coming back. Results confirmed that B7-H4 mRNA and protein levels were abnormally overexpressed on the samples of recurrence TNBC patients after neoadjuvant chemotherapy treatment (Fig. $3 a-c)$. In this data set, Kaplan-Meier analysis of the local recurrence-free survival between the different groups (higher versus lower expression) showed that women who had higher B7-H4 expression had a significantly increased risk of local recurrence, even after neoadjuvant chemotherapy (Fig. 3d). Especially, when the cohort was divided into Dritter, the increasing rates of local recurrence were noted as the intensities of $\mathrm{B} 7-\mathrm{H} 4$ expression rose (Fig. 3e). In univariate analysis, recurrence-free survival was significantly worse in tumors from patients diagnosed with TNBC which displayed the top strong staining intensity $\mathrm{B} 7-\mathrm{H} 4$ expression $(\mathrm{HR}=0.256,95 \% \mathrm{CI} 0.103-0.635, p=0.003)$. This is of great clinical significance as disease recurrence in patients diagnosed with cancers of TNBC.

\section{Anti-proliferative effect of B7-H4 mAb} on the chemo-resistant TNBC cell lines

Previous results suggested the potential functions of $\mathrm{B} 7-\mathrm{H} 4$ involved in the tumorigenesis and progression 

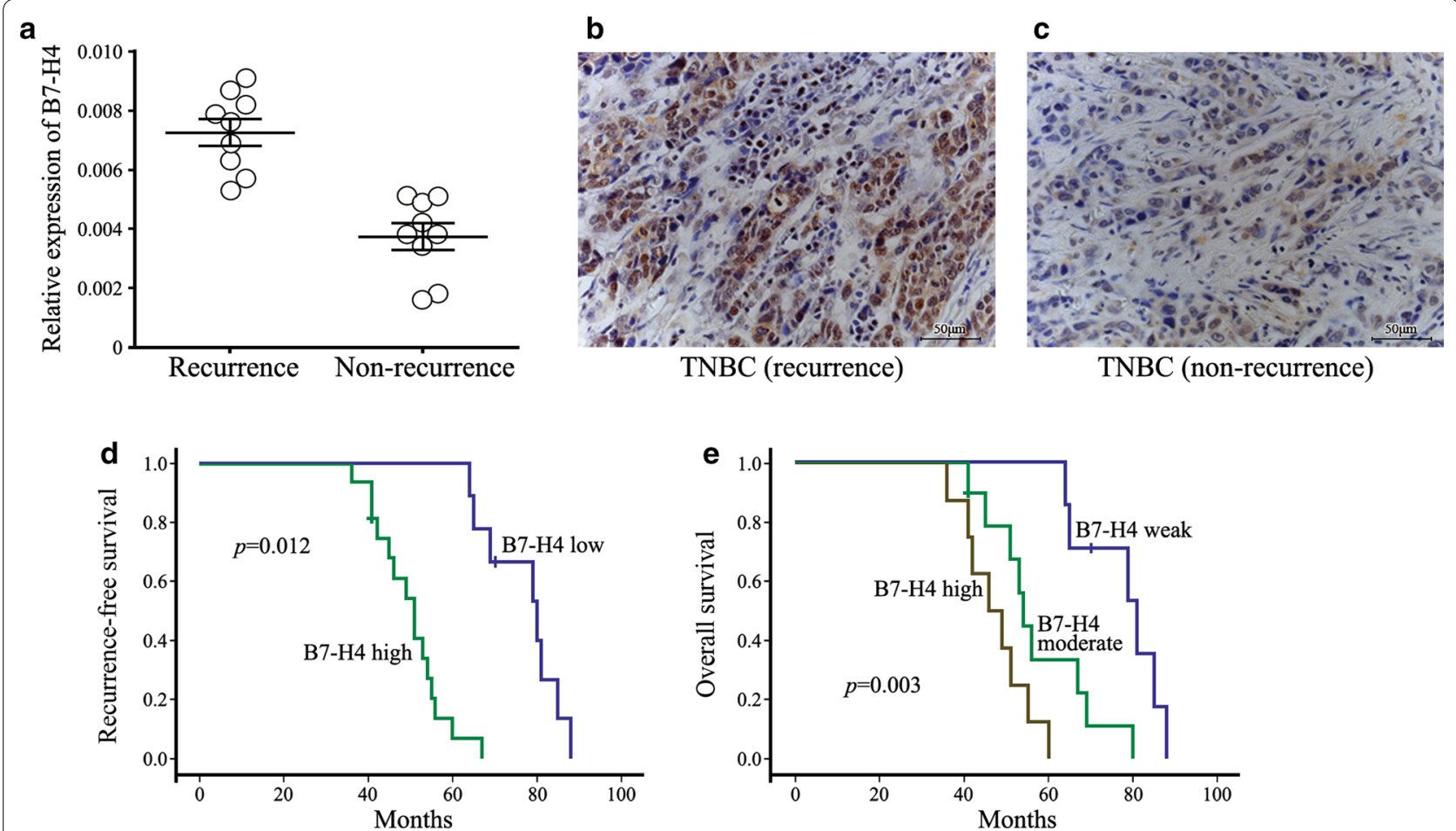

Fig. 3 B7-H4 is aberrantly overexpressed in TNBC recurrence patients after neoadjuvant chemotherapy treatment. a The expression level of B7-H4 mRNA in the samples of recurrence TNBC patients compared with non-recurrence TNBC tissue samples. $\mathbf{b}$ B7-H4 immunostaining in the samples of recurrence TNBC patients compared with non-recurrence TNBC tissue samples (c). $\mathbf{d}$ Kaplan-Meier plots of overall survival in recurrence TNBC patients stratified by B7-H4 expression levels. e Kaplan-Meier plots of overall survival in recurrence TNBC patients stratified by B7-H4 expression intensity

of TNBC, even in the recurrence stage, which led us suspect its potential drug resistance capability in the chemo-treatment. Therefore, DOX-resistant MDAMB-435 cells (MDA-MB-435/DOX) and the parental MDAMB-435/WT cells were plated in 96-well plates at $1 \times 10^{4}$ cells/well and treated with B7-H4 mAb MIH43 at various doses (range $1-10 \mu \mathrm{g} / \mathrm{mL}$ ) and compared to treatment with isotype control mAb IgG1. Within the studied concentration range, both $1 \mu \mathrm{g} / \mathrm{mL}$ and $5 \mu \mathrm{g} /$ $\mathrm{mL} \mathrm{mAb}$ MIH43 did not show any cytotoxic effects on MDA-MB-435/DOX cells following $24 \mathrm{~h}$ incubation, whereas $5 \mu \mathrm{g} / \mathrm{mL}$ mAb MIH43 was shown to be cytotoxic for MDAMB-435/WT cells. Within the tested incubation time prolonged, mAb MIH43 was shown to be more cytotoxic to MDAMB-435/WT cells than to MDA-MB-435/DOX cells upon 48 and $72 \mathrm{~h}$ incubation. Monotherapy with mAb MIH43 $(10 \mu \mathrm{g} / \mathrm{mL})$ resulted in over $40 \%$ growth inhibition of MDAMB-435/WT cells, and nearly $25 \%$ inhibition of MDA-MB-435/DOX cell growth at $48 \mathrm{~h}$ (Fig. $4 \mathrm{a}-\mathrm{c}$ ).

In order to study the possible role of $\mathrm{B} 7-\mathrm{H} 4$ in affecting the sensitivity of TNBC cells to DOX, paclitaxel or carboplatin, MDAMB-435/WT and MDA-MB-435/
DOX cells were treated with these chemo-drugs together with mAb MIH43 $(10 \mu \mathrm{g} / \mathrm{mL})$ for $48 \mathrm{~h}$. Results showed that treatment of mAb MIH43 with could enhance the sensitivity of both two cell lines to paclitaxel in a significant manner. Similar results were observed for the carboplatin and DOX treatment (Fig. 4d-f).

\section{B7-H4 silencing increases doxorubicin sensitivity by PTEN/ PI3K/AKT pathway}

Dox is well known for exerting their cytotoxic effects through induction of apoptosis [21, 22], and hence, we investigated whether the increased DOX cytotoxicity observed in B7-H4 knockdown cells could be related to effects on apoptosis. The extent of apoptosis was investigated by measuring DNA fragmentation by apoptosis-specific ELISA detection kit. As shown in Fig. 5a, apoptosis-specific ELISA detection revealed that a dose-dependent of apoptosis was observed in MDAMB-435/WT cells and the B7-H4 knockdown cells were about 1.5 -fold more sensitive to DOX than parental and control cells. Statistical analysis showed that the differences between B7-H4 knockdown and control cells 


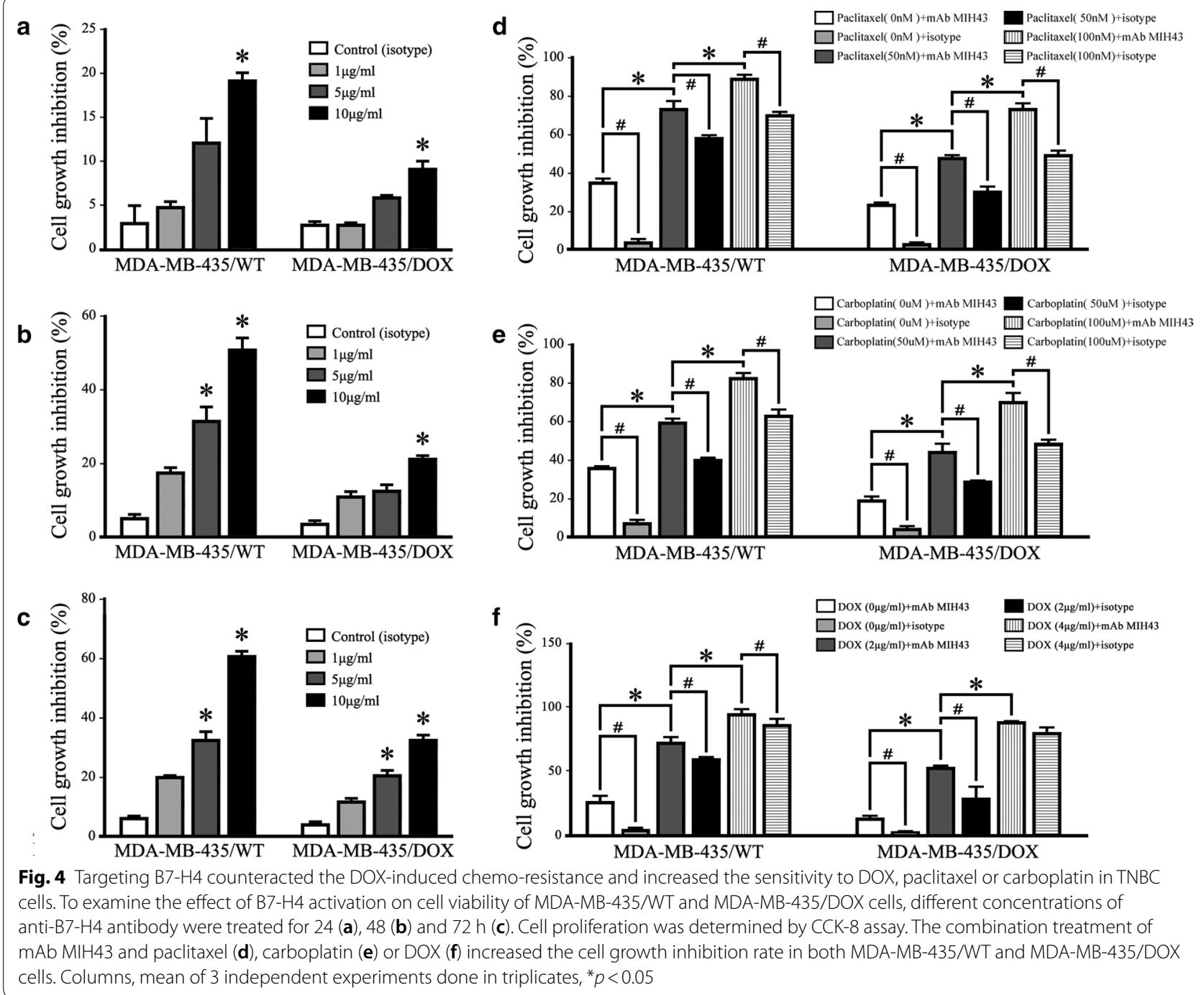

were significant. These results indicate that $\mathrm{B} 7-\mathrm{H} 4$ plays a role in tumor cell apoptosis induced by DOX. To elucidate further the effect of $\mathrm{B} 7-\mathrm{H} 4$ on DOX resistance, MDA-MB-435/WT cells and B7-H4 knockdown cells were treated with $5 \mu \mathrm{g} / \mathrm{mL}$ DOX for $72 \mathrm{~h}$, we observed dramatically increased amounts of cleaved-PARP, cleaved-Caspase-3, cleaved-Caspase-7 and cleavedCaspase-9 fragmentations in B7-H4 siRNA-transfected cells than in control MDA-MB-435/WT cells (Fig. 5b).

Previous studies have demonstrated that B7-H4 activation leads to abnormally the down-regulation of the AKT pathway in EBV-positive B-cell lymphoma cells [23]. The physiological function of phosphatase and tensin homologue (PTEN), a lipid phosphatase, is a frequently mutated tumor suppressor gene that opposes the PI3K/AKT pathway through dephosphorylation of phosphoinositide-3,4,5-triphosphate [24, 25]. Therefore, we investigate whether B7-H4 was involved by targeting PTEN through the PI3K/Akt signaling pathway. As seen in Fig. 5c, the silencing of B7-H4 induced a dramatic increase in the expression of PTEN both in untreated and DOX-treated cells. Furthermore, the phosphorylation level of p-PI3K was dramatically repressed in B7-H4 silenced cells with reduced Akt phosphorylation (Fig. 5c).

To validate this conclusion, we evaluated whether overexpression of B7-H4 was sufficient to activate PI3K/Akt pathway. B7-H4 cDNA was transiently transfected into B7-H4 knockdown cells 435-siB7-H4 (Fig. 5c, right) and then the phosphorylation level of both PI3K and Akt increased with the B7-H4 expression, further confirming an important role of $\mathrm{B} 7-\mathrm{H} 4$ in regulating the PI3K/Akt signaling pathway. 


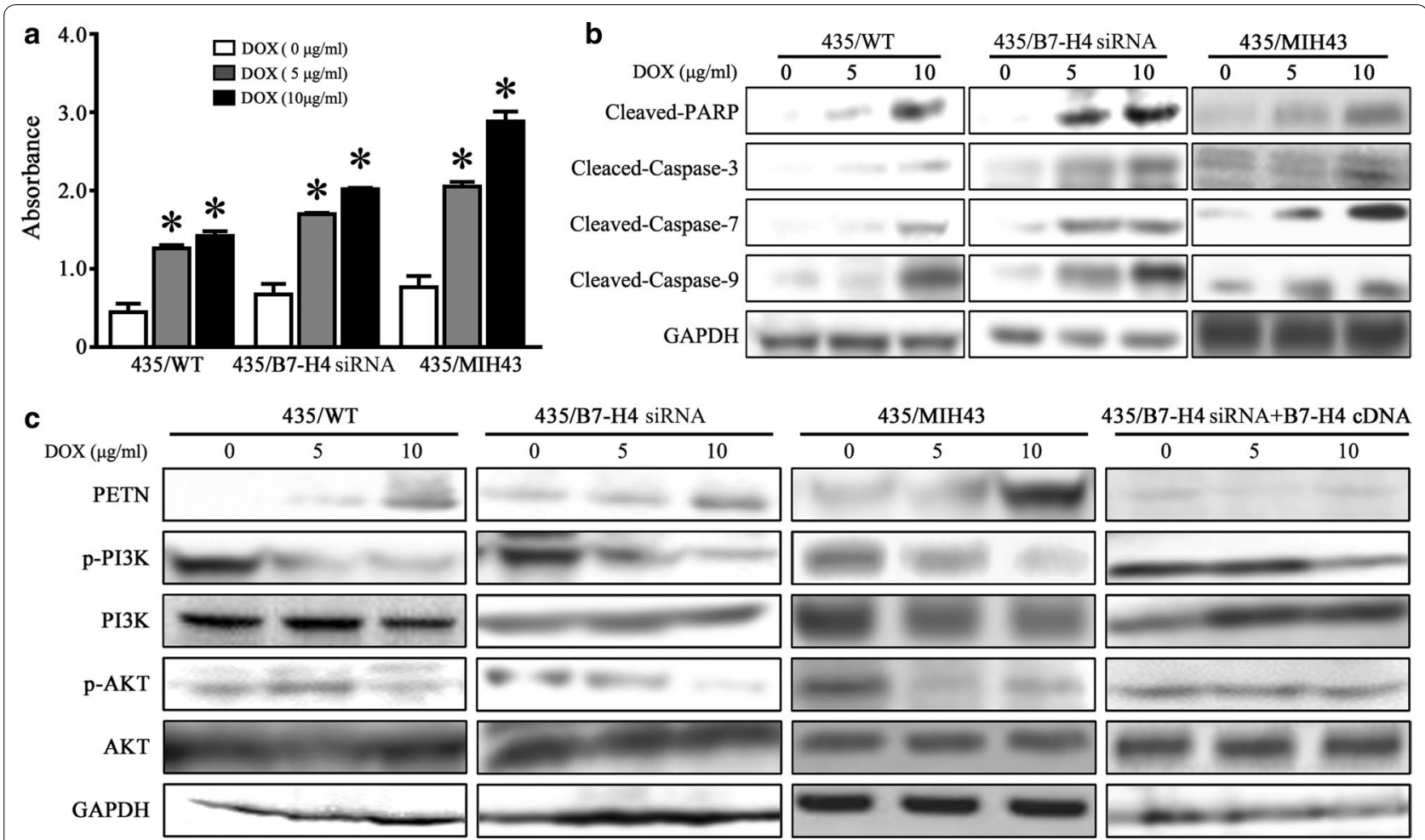

Fig. 5 a B7-H4 silencing or treatment of $\mathrm{mAb}$ MIH43 sensitized breast cancer cells to DOX-induced apoptosis. The percentage of apoptotic cells was investigated by measuring DNA fragmentation by apoptosis-specific ELISA detection kit. b B7-H4 silencing or treatment of mAb MIH43 increased apoptosis via promoting cleaved-PARP, cleaved-Caspase-3, cleaved-Caspase-7 and cleaved-Caspase-9 fragmentations. c Knockdown of $\mathrm{B} 7-\mathrm{H} 4$ or treatment of $\mathrm{mAb} \mathrm{MIH43}$ enhanced the level of PTEN and abolished the phosphorylation level of PI3K and AKT, whereas overexpression of B7-H4 could counteract these effects

\section{Discussion}

Evading the antitumor immunity is crucial for the development and progression of cancer [26, 27]. Understanding the dynamic interaction between tumors and the immune system is impendent for the advance of a new cancer immunotherapy [28, 29]. B7-H4 was identified to be one of the fellow B7 family members in 2003, and shown to bind a currently unknown receptor(s) on activated $\mathrm{T}$ cells thus resulting in inhibition of $\mathrm{T}$ cell effector function in vitro $[13,30]$. B7-H4 mRNA is widely expressed in nonlymphoid tissues [31], but its protein expression is largely absent in most normal human somatic tissues, with the exception of epithelial cells from the female genital tract, lung, kidney and pancreas [30]. Abnormal overexpression of B7-H4 was reported in a variety of malignancies including lung, ovarian, breast, prostate, and esophageal cancers [32-36]. B7-H4 is a ligand within the B7 family that has been implicated as a negative regulator of T-cell-mediated immunity, and its expression was inversely related the number of tumor infiltrating $\mathrm{T}$ cells $[37,38]$. Although the mechanism is still not clear, it is proposed that B7-H4 might employ to evade the host immune system by regulating the differentiation of $\mathrm{T}$ cells. The most current literature supports that $\mathrm{B} 7-\mathrm{H} 4$ is a potential negative prognostic indicator for many tumors and could aid in transforming pre-cancerous cells and then protecting them from immunosurveillance [39]. However, in Rahba's study, he found that reduced MHC I expression and granzyme $\mathrm{B}$ expression in $\mathrm{CD}^{+} \mathrm{T}$ cells infiltrating tumors were detected in $\mathrm{B} 7-\mathrm{H} 4^{-1-}$ background mice, together with other evidence showed that B7-H4 expression was not necessary for tumor development but could limit the mammary tumor growth $[34,40]$. Collectively, the role of $\mathrm{B} 7-\mathrm{H} 4$ in immune evasion in the cancer microenvironment is yet to be elucidated, especially in breast cancer. Therefore, we want to further study the expression pattern of B7-H4 in TNBC and its potential mechanism.

In this study, we used Cancer Genome Atlas Microarray Database to predict that $\mathrm{B} 7-\mathrm{H} 4$ transcript was dominantly increased in the subtype of invasive breast carcinoma. In the following study, we found that B7-H4 expression was elevated in TNBC cell lines and TNBC patients compared with non-TNBC cells and adjacent non-tumor tissues breast cancer patients and that increased $\mathrm{B} 7-\mathrm{H} 4$ expression was associated with 
advanced TNM stage and the tendency of metastasis and recurrence. In addition, there was a reverse correlation between the expression of $\mathrm{B} 7-\mathrm{H} 4$ and $\mathrm{AR}$ and the outcome of patients. These findings indicate that B7-H4 expression might be linked to more aggressive subtypes of breast cancer and are consistent with previous studies reporting that $\mathrm{B} 7-\mathrm{H} 4$ expression is associated with a poor prognosis in oral squamous cell carcinoma [41], non-small cell lung cancer [42], renal cell carcinoma [43], and glioma [44]. TNBC is a very aggressive subtype of breast cancer due to its lack of the hormonal receptors as well as HER-2 and thus unresponsive to hormonal therapies such as ER/PR antagonists or trastuzumab therapies. Till now, chemotherapy is still the first line treatment of TNBC, however, resistance, relapse, poor response rate and toxicity are common companies associated with chemotherapeutic drugs. Although Leong et al. [45] devised an antibody-drug conjugates against $\mathrm{B} 7-\mathrm{H} 4$ to treat patient-derived xenograft models of triple-negative breast cancer, however, whether chemotherapy could upregulate B7-H4 upregulation and lead to cancer cells become drug-resistant have not been completely studied and reported yet. In our study, we found that B7-H4 expression levels were abnormally overexpressed on the samples of recurrence TNBC patients after neoadjuvant chemotherapy treatment, which indicated that neoadjuvant chemotherapy might induce B7-H4 upregulation. And the increasing rates of local recurrence were noted as the intensities of B7-H4 expression strengthened. Therefore, it might be highly suggested that blocking B7-H4 could be an alternative to to increase the efficacy and reduce the toxicity of the chemotherapeutic drugs.

B7-H4 is a well-defined transmembrane protein, containing one signal peptide and hydrophobic transmembrane domain uniquely anchored to the cell membrane via a GPI linkage $[13,46,47]$. However, some tumor cells were shown to express $\mathrm{B} 7-\mathrm{H} 4$ protein in different subcellular distributions $[48,49]$. In our study, we found that B7-H4 protein was shown intense circumferential membranous and cytoplasmic expression in most TNBC cells. So far, the mechanisms and functional implications of B7-H4 subcellular localization remain unclear. Therefore, we selected typically monoclonal antibody MIH43 of $\mathrm{B} 7-\mathrm{H} 4$ for detecting its potential functions. Among the selected TNBC cell lines, the human MDA-MB-435 cell line used in our study was testified to have the highest B7-H4 expression and closely similar to those with MDA-MB-231 and MDAMB-468 breast cancer cells [50, 51]. In this study, we examined the role of $\mathrm{B} 7-\mathrm{H} 4$ in doxorubicin resistance of TNBC cells. Results showed that mAb MIH43 resulted in increased cell growth inhibition of DOX-resistant MDA-MB-435 cells and the parental
MDAMB-435/WT cells. In addition, treatment of mAb MIH43 with could enhance the sensitivity of both two cell lines to paclitaxel in a significant manner. Our findings show that targeting B7-H4 could counteract cellular resistance to doxorubicin, and increase the sensitivity to paclitaxel in TNBC cells. Furthermore, in attempts to elucidate the mechanisms underlying the observed effects, we obtained the evidence from a series of functional experiments.

In order to completely elucidate the function of $\mathrm{B} 7-\mathrm{H} 4$, the knockdown and overexpression of the $\mathrm{B} 7-\mathrm{H} 4$ plasmids were constructed respectively. Our finding showed that the apoptotic rate was increased 1.5-fold of TNBC cells sensitive to doxorubicin when B7-H4 was knockdown. Previous investigations showed that breast cancer with axillary lymph node metastases was associated with the abnormal suppressor gene PTEN, which could play a negative regulatory role in the PI3K/Akt signaling pathway $[52,53]$. Furthermore, Basho et al. [54] reported that the PI3K/Akt pathway is the major frequently dysregulated pathways in TNBC, which favors the metaplastic TNBC for its transcriptional profiling as the mesenchymal subtype. Interestingly, we found that downregulation of $\mathrm{B} 7-\mathrm{H} 4$ restored the expression of PTEN and reduced phosphorylation of both PI3K and AKT, whereas overexpression of B7-H4 activated PI3K/AKT signaling. This may explain why the B7-H4 knockdown TNBC cells became more prone to doxorubicin-induced apoptosis, and the findings are in accordance with reports showing induction of apoptosis following a blockade of PTEN signaling in multiple cancers [55-57]. On the basis of these findings, we investigated the effects of B7-H4 knockdown on PTEN/PI3K/Akt-regulated genes involved in mitochondrial-pathway apoptosis. The increased expression of cleaved-PARP, cleaved-Caspase-3, cleaved-Caspase-7 and cleaved-Caspase- 9 fragmentations in B7-H4 siRNA-transfected cells than in control MDAMB-435/WT cells, whereas the effects could be counteracted by overexpression of $\mathrm{B} 7-\mathrm{H} 4$.

\section{Conclusion}

Our study investigating the role of B7-H4 in TNBC development and progression showed that $\mathrm{B} 7-\mathrm{H} 4$ was highly expressed in TNBC patients and cells and was associated with a poor prognosis and metastasis. Furthermore, our data indicated that neutralizing antibody against B7-H4 significantly decreased tumor cell viability in vitro, and the protein confers resistance to doxorubicin by reducing the sensitivity of breast cancer cells to apoptosis, mediated via the PTEN/PI3K/Akt pathway. Collectively, these findings provide new insight into the role of B7-H4 in TNBC, which might serve as a prognostic biomarker indicative of poor outcomes and be an effective therapeutic target of TNBC treatment. 


\section{Additional files}

Additional file 1: Figure S1. (A, B) The effects of B7-H4 knockdown and overexpression was confirmed by real-time PCR and western blotting, respectively. (C) The growth inhibition was influenced after B7-H4 downregulation in MDA-MB-435 WT and MDA-MB-435/DOX cells, respectively.

Additional file 2: Figure S2. (A, B) The expression level of B7-H4 protein in various breast cancer cell lines was evaluated using western blotting. The data are presented as the mean \pm SD

\section{Authors' contributions}

CY collected clinical samples and their information. XBL and LW performed the cell experiments in vitro. LW contributed to the design of the experiments and analyze all the data. FBK helped design the experiment, provided materials and revised the manuscript. All authors read and approved the final manuscript.

\section{Author details}

1 Department of Orthopedic Oncology, the Third Hospital of Hebei Medical University, Shijiazhuang, Hebei, People's Republic of China. ${ }^{2}$ Department of General Surgery, the Fourth Hospital of Hebei Medical University, Shijiazhuang, Hebei, People's Republic of China. ${ }^{3}$ Department of Thoracic Surgery, the Fourth Hospital of Hebei Medical University, Shijiazhuang, Hebei, People's Republic of China. ${ }^{4}$ Department of Pathology, the Fourth Hospital of Shijiazhuang, Shijiazhuang, China. ${ }^{5}$ Department of Liver Diseases, Bethune International Peace Hospital, Shijiazhuang, Hebei, People's Republic of China.

\section{Acknowledgements}

We appreciated that Dr. Yu Zhao provided the technique support during the immunohistochemistry.

\section{Competing interests}

The authors declare that they have no competing interests.

\section{Availability of data and materials}

All the data and material could be traced from the paper we have published before.

\section{Consent for publication}

All the listed authors have participated actively in the study, and have seen and approved the submitted manuscript.

\section{Ethics approval and consent to participate}

Tissue samples (frozen and FFPE) were separately obtained from 65 untreated Chinese women diagnosed with TNBC at the Fourth Hospital of Hebei Medical University from January 2005 to December 2010. This study was reviewed and approved by the Review Board of Fourth Hospital of Hebei Medical University. All patients gave their written and informed consent.

\section{Funding}

This work was supported by several Grants from National Natural Science Foundation of China (81402228, 81772858), Hebei Natural Science Foundation (H2015206216), HeBei Province Education Foundation (QN2014049) and HeBei Province Medical Foundation (ZL20140334, 20160649).

\section{Publisher's Note}

Springer Nature remains neutral with regard to jurisdictional claims in published maps and institutional affiliations.

Received: 24 February 2018 Accepted: 6 July 2018

Published online: 13 July 2018

\section{References}

1. Fleisher B, Clarke C, Ait-Oudhia S. Current advances in biomarkers for targeted therapy in triple-negative breast cancer. Breast Cancer. 2016;8:183-97.

2. Yao H, He G, Yan S, Chen C, Song L, Rosol TJ, Deng X. Triple-negative breast cancer: is there a treatment on the horizon? Oncotarget. 2016;8(1):1913.

3. Bianchini G, Balko JM, Mayer IA, Sanders ME, Gianni L. Triple-negative breast cancer: challenges and opportunities of a heterogeneous disease. Nat Rev Clin Oncol. 2016;13(11):674-90.

4. Saha P, Nanda R. Concepts and targets in triple-negative breast cancer: recent results and clinical implications. Ther Adv Med Oncol. 2016:8(5):351-9.

5. Gu G, Dustin D, Fuqua SA. Targeted therapy for breast cancer and molecular mechanisms of resistance to treatment. Curr Opin Pharmacol. 2016;31:97-103.

6. Wali VB, Langdon CG, Held MA, Platt JT, Patwardhan GA, Safonov A, Aktas B, Pusztai L, Stern DF, Hatzis C. Systematic drug screening identifies tractable targeted combination therapies in triple-negative breast cancer. Cancer Res. 2016;77(2):566-78.

7. Chacon JA, Schutsky K, Powell DJ. The impact of chemotherapy, radiation and epigenetic modifiers in cancer cell expression of immune inhibitory and stimulatory molecules and anti-tumor efficacy. Vaccines. 2016;4(4):43.

8. Wang L, Kang FB, Shan BE. B7-H3-mediated tumor immunology: friend or foe? Int J Cancer. 2014;134(12):2764-71.

9. Lee V, Murphy A, Le DT, Diaz LA Jr. Mismatch repair deficiency and response to immune checkpoint blockade. Oncologist. 2016;21(10):1200-11.

10. Tsiatas M, Grivas P. Immunobiology and immunotherapy in genitourinary malignancies. Ann Transl Med. 2016;4(14):270.

11. Flies DB, Chen L. The new B7s: playing a pivotal role in tumor immunity. J Immunother. 2007;30(3):251-60.

12. Greenwald RJ, Freeman GJ, Sharpe AH. The B7 family revisited. Annu Rev Immunol. 2005;23:515-48.

13. Sica GL, Choi IH, Zhu G, Tamada K, Wang SD, Tamura H, Chapoval Al, Flies DB, Bajorath J, Chen L. B7-H4, a molecule of the B7 family, negatively regulates T cell immunity. Immunity. 2003;18(6):849-61.

14. Crispen PL, Boorjian SA, Lohse CM, Leibovich BC, Kwon ED. Predicting disease progression after nephrectomy for localized renal cell carcinoma: the utility of prognostic models and molecular biomarkers. Cancer. 2008;113(3):450-60.

15. Smith JB, Stashwick C, Powell DJ Jr. B7-H4 as a potential target for immunotherapy for gynecologic cancers: a closer look. Gynecol Oncol. 2014;134(1):181-9.

16. Maskey N, Li K, Hu M, Xu Z, Peng C, Yu F, Cao H, Chen J, Li Y, Yang G. Impact of neoadjuvant chemotherapy on lymphocytes and co-inhibitory B7-H4 molecule in gastric cancer: low B7-H4 expression associates with favorable prognosis. Tumour Biol. 2014;35(12):11837-43.

17. Abadi YM, Jeon H, Ohaegbulam KC, Scandiuzzi L, Ghosh K, Hofmeyer KA, Lee JS, Ray A, Gravekamp C, Zang X. Host b7x promotes pulmonary metastasis of breast cancer. J Immunol. 2013;190(7):3806-14.

18. Tan PH, Ellis IO. Myoepithelial and epithelial-myoepithelial, mesenchymal and fibroepithelial breast lesions: updates from the WHO classification of tumours of the breast 2012. J Clin Pathol. 2013;66(6):465-70.

19. Falamarzian A, Aliabadi HM, Molavi O, Seubert JM, Lai R, Uludag H, Lavasanifar A. Effective down-regulation of signal transducer and activator of transcription 3 (STAT3) by polyplexes of siRNA and lipid-substituted polyethyleneimine for sensitization of breast tumor cells to conventional chemotherapy. J Biomed Mater Res Part A. 2014;102(9):3216-28.

20. Yang TJ, Morrow M, Modi S, Zhang Z, Krause K, Siu C, McCormick B, Powell SN, Ho AY. The Effect of molecular subtype and residual disease on locoregional recurrence in breast cancer patients treated with neoadjuvant chemotherapy and postmastectomy radiation. Ann Surg Oncol. 2015:22(Suppl 3):S495-501.

21. Zhang H, Guo Y, Song Y, Shang C. Long noncoding RNA GAS5 inhibits malignant proliferation and chemotherapy resistance to doxorubicin in bladder transitional cell carcinoma. Cancer Chemother Pharmacol. 2017;79(1):49-55.

22. Zhao Y, Huan ML, Liu M, Cheng Y, Sun Y, Cui H, Liu DZ, Mei QB, Zhou SY. Doxorubicin and resveratrol co-delivery nanoparticle to overcome doxorubicin resistance. Sci Rep. 2016;6:35267. 
23. Park GB, Song H, Kim YS, Sung M, Ryu JW, Lee HK, Cho DH, Kim D, Lee WJ, Hur DY. Cell cycle arrest induced by engagement of B7-H4 on Epstein-Barr virus-positive B-cell lymphoma cell lines. Immunology. 2009;128(3):360-8.

24. Maddika S, Kavela S, Rani N, Palicharla VR, Pokorny JL, Sarkaria JN, Chen J. WWP2 is an E3 ubiquitin ligase for PTEN. Nat Cell Biol. 2011;13(6):728-33.

25. Song MS, Carracedo A, Salmena L, Song SJ, Egia A, Malumbres M, Pandolf PP. Nuclear PTEN regulates the APC-CDH1 tumor-suppressive complex in a phosphatase-independent manner. Cell. 2011;144(2):187-99.

26. Serrels A, Frame MC. FAK goes nuclear to control antitumor immunity-a new target in cancer immuno-therapy. Oncoimmunology. 2016;5(4):e1119356.

27. Sugie T, Toi M. Antitumor immunity and advances in cancer immunotherapy. Breast Cancer. 2017;24(1):1-2.

28. Zanetti M. A second chance for telomerase reverse transcriptase in anticancer immunotherapy. Nat Rev Clin Oncol. 2016;14(2):115.

29. Sukari A, Nagasaka M, Al-Hadidi A, Lum LG. Cancer immunology and immunotherapy. Anticancer Res. 2016;36(11):5593-606

30. Choi IH, Zhu G, Sica GL, Strome SE, Cheville JC, Lau JS, Zhu Y, Flies DB, Tamada K, Chen L. Genomic organization and expression analysis of B7-H4, an immune inhibitory molecule of the B7 family. J Immunol. 2003;171(9):4650-4

31. Yi KH, Chen $\mathrm{L}$. Fine tuning the immune response through $\mathrm{B} 7-\mathrm{H} 3$ and B7-H4. Immunol Rev. 2009;229(1):145-51.

32. Zhang $X$, Cai L, Zhang G, Shen Y, Huang J. B7-H4 promotes tumor growth and metastatic progression in lung cancer by impacting cell proliferation and survival. Oncotarget. 2017;8(12):18861.

33. Liang L, Jiang Y, Chen JS, Niu N, Piao J, Ning J, Zu Y, Zhang J, Liu J. B7-H4 expression in ovarian serous carcinoma: a study of 306 cases. Hum Pathol. 2016;57:1-6.

34. Rahbar R, Lin A, Ghazarian M, Yau HL, Paramathas S, Lang PA, Schildknecht A, Elford AR, Garcia-Batres C, Martin B, et al. B7-H4 expression by nonhematopoietic cells in the tumor microenvironment promotes antitumor immunity. Cancer Immunol Res. 2015;3(2):184-95.

35. Mukhopadhyay A, Khoury T, Stein L, Shrikant P, Sood AK. Prostate derived Ets transcription factor and carcinoembryonic antigen related cell adhesion molecule 6 constitute a highly active oncogenic axis in breast cancer. Oncotarget. 2013;4(4):610-21.

36. Wang L, Cao NN, Wang S, Man HW, Li PF, Shan BE. Roles of coinhibitory molecules B7-H3 and B7-H4 in esophageal squamous cell carcinoma. Tumour Biol. 2016;37(3):2961-71.

37. Liang M, Li J, Wang D, Li S, Sun Y, Sun T, Zhang J, Chen X, Li Q, Sun S. T-cell infiltration and expressions of T lymphocyte Co-inhibitory B7-H1 and B7-H4 molecules among colorectal cancer patients in northeast China's Heilongjiang province. Tumour Biol. 2014;35(1):55-60.

38. Zhao LW, Li C, Zhang RL, Xue HG, Zhang FX, Zhang F, Gai XD. B7-H1 and B7-H4 expression in colorectal carcinoma: correlation with tumor FOXP3(+) regulatory T-cell infiltration. Acta Histochem. 2014;116(7):1163-8.

39. Fauci JM, Straughn JM Jr, Ferrone S, Buchsbaum DJ. A review of B7-H3 and B7-H4 immune molecules and their role in ovarian cancer. Gynecol Oncol. 2012;127(2):420-5.

40. Rahbar R, Ohashi PS. B7-H4 is a positive regulator of antitumor immunity. Oncoimmunology. 2016;5(1):e1050575.

41. Wu L, Deng WW, Yu GT, Mao L, Bu LL, Ma SR, Liu B, Zhang WF, Sun ZJ. B7-H4 expression indicates poor prognosis of oral squamous cell carcinoma. Cancer Immunol Immunother. 2016;65(9):1035-45.
42. Wang W, Xu C, Wang Y, Yu L, Zhang X. Prognostic values of B7-H4 in nonsmall cell lung cancer. Biomarkers. 2016;21:1-16.

43. Fukuda T, Kamai T, Masuda A, Nukui A, Abe H, Arai K, Yoshida K. Higher preoperative serum levels of PD-L1 and B7-H4 are associated with invasive and metastatic potential and predictable for poor response to VEGFtargeted therapy and unfavorable prognosis of renal cell carcinoma. Cancer Med. 2016:5(8):1810-20.

44. Yao Y, Ye H, Qi Z, Mo L, Yue Q, Baral A, Hoon DS, Vera JC, Heiss JD, Chen CC, et al. B7-H4(B7X)-mediated cross-talk between glioma-initiating cells and macrophages via the IL6/JAK/STAT3 pathway lead to poor prognosis in glioma patients. Clin Cancer Res. 2016;22(11):2778-90.

45. Leong SR, Liang WC, Wu Y, Crocker L, Cheng E, Sampath D, Ohri R, Raab $\mathrm{H}$, Hass PE, Pham T, et al. An anti-B7-H4 antibody-drug conjugate for the treatment of breast cancer. Mol Pharm. 2015;12(6):1717-29.

46. Zang X, Loke P, Kim J, Murphy K, Waitz R, Allison JP. B7x: a widely expressed B7 family member that inhibits T cell activation. Proc Natl Acad Sci USA. 2003;100(18):10388-92

47. Prasad DV, Richards S, Mai XM, Dong C. B7S1, a novel B7 family member that negatively regulates T cell activation. Immunity. 2003;18(6):863-73.

48. Quandt D, Fiedler E, Boettcher D, Marsch W, Seliger B. B7-h4 expression in human melanoma: its association with patients'survival and antitumor immune response. Clin Cancer Res. 2011;17(10):3100-11.

49. Miyatake T, Tringler B, Liu W, Liu SH, Papkoff J, Enomoto T, Torkko KC, Dehn DL, Swisher A, Shroyer KR. B7-H4 (DD-O110) is overexpressed in high risk uterine endometrioid adenocarcinomas and inversely correlated with tumor T-cell infiltration. Gynecol Oncol. 2007;106(1):119-27.

50. Sellappan S, Grijalva R, Zhou X, Yang W, Eli MB, Mills GB, Yu D. Lineage infidelity of MDA-MB-435 cells: expression of melanocyte proteins in a breast cancer cell line. Can Res. 2004;64(10):3479-85.

51. Chambers AF. MDA-MB-435 and M14 cell lines: identical but not M14 melanoma? Can Res. 2009:69(13):5292-3.

52. Wang LL, Hao S, Zhang S, Guo LJ, Hu CY, Zhang G, Gao B, Zhao JJ, Jiang Y, Tian WG, et al. PTEN/PI3K/AKT protein expression is related to clinicopathologic features and prognosis in breast cancer with axillary lymph node metastases. Hum Pathol. 2016;61:49-57.

53. Ebbesen SH, Scaltriti M, Bialucha CU, Morse N, Kastenhuber ER, Wen HY, Dow LE, Baselga J, Lowe SW. Pten loss promotes MAPK pathway dependency in HER2/neu breast carcinomas. Proc Natl Acad Sci USA. 2016;113(11):3030-5

54. Basho RK, Gilcrease M, Murthy RK, Helgason T, Karp DD, Meric-Bernstam F, Hess KR, Herbrich SM, Valero V, Albarracin C, et al. Targeting the PI3K/ AKT/mTOR pathway for the treatment of mesenchymal triple-negative breast cancer: evidence from a phase 1 trial of mTOR inhibition in combination with liposomal doxorubicin and bevacizumab. JAMA Oncol. 2016;3(4):509-15.

55. Ryu JW, Sik Choe S, Ryu SH, Park EY, Wook Lee B, Keun Kim T, Hoon Ha C, Lee SW. Paradoxical induction of growth arrest and apoptosis by EGF via the up-regulation of PTEN by activating Redox factor-1/Egr-1 in human lung cancer cells. Oncotarget. 2016;8(3):4181.

56. Wang SQ, Wang C, Chang LM, Zhou KR, Wang JW, Ke Y, Yang DX, Shi HG, Wang $\mathrm{R}$, Shi $\mathrm{XL}$, et al. Geridonin and paclitaxel act synergistically to inhibit the proliferation of gastric cancer cells through ROS-mediated regulation of the PTEN/PI3K/Akt pathway. Oncotarget. 2016;7(45):72990-3002.

57. Nip H, Dar AA, Saini S, Colden M, Varahram S, Chowdhary H, Yamamura S, Mitsui Y, Tanaka Y, Kato T, et al. Oncogenic microRNA-4534 regulates PTEN pathway in prostate cancer. Oncotarget. 2016;7(42):68371-84. 